\title{
Diffuse Alveolar Hemorrhage after Anabolic Steroid Use: A Case Report
}

\author{
Gómez Rodríguez B* and Rodríguez Portal JA
}

Hospital Universitario Virgen del Rocio, Seville, Spain

\begin{abstract}
Introduction: Corticosteroids have an important role in multiples physiological mechanisms, including carbohydrate metabolism, protein catabolism and a potent anti-inflammatory activity that made them basic to the treatment of a large number of autoimmune diseases. Anabolic steroid treatment has been associated with a large number of side effects: Impaired hepatic function, hypotrophic hypogonadism, cardiac involvement, thrombosis development, etc. However, there are few cases reported in the medical literature of diffuse alveolar haemorrhage secondary to this cause.
\end{abstract}

In recent years there has been an increase in the use of corticosteroids in an abusive way for non-medical purposes, specifically in the field of bodybuilding, with the aim of gaining muscle mass quickly and which is worrying as well as the adverse effects previously mentioned, by the disconcerting of the long-term adverse effects.

Presentation of the Case: We present the case of a young male bodybuilder patient who presented diffuse alveolar haemorrhage due to several cycles of anabolic steroid treatment. The clinical picture began with dyspnea and hemoptysis associated with an X-ray and chest CT with compatible infiltrates. The patient presented a favorable response to the administration of corticosteroids in high doses and after the withdrawal of the anabolic steroids the normalization of the imaging tests took place, without presenting subsequent alterations in the pulmonary function.

Conclusions: We consider that it is important to keep in mind the diffuse alveolar haemorrhage as a complication associated with the use of anabolic steroids and it must be taken into account that the patient is precocious with dyspnea and hemoptysis.

Keywords: Diffuse alveolar haemorrhage; Anabolic steroids; Adverse effects

\section{Introduction}

Corticosteroids have an important role in multiples physiological mechanisms, including carbohydrate metabolism, protein catabolism and a potent anti-inflammatory activity that made them basic to the treatment of a large number of autoimmune diseases.

The treatment with anabolic steroids has been related to a large number of side effects at different organs and systems: kidney $[1,2]$, adenomas and hepatic haemorrhages [3] myocardial infarction [4], cerebral haemorrhage [5] and thromboembolic complications [6]. However, there are only two previous cases described in the medical literature that relate the abuse of anabolic steroids and the development of diffuse alveolar haemorrhage [7].

In recent years there has been an increase in the use of corticosteroids in an abusive way for non-medical purposes, specifically in the field of bodybuilding, with the aim of gaining muscle mass quickly and which is worrying as well as the adverse effects previously mentioned, by the disconcerting of the long-term adverse effects [8].

\section{Clinical Case}

Our patient was a 24-year-old male admitted for multiple trauma secondary to traffic accident with open tibial fracture stabilized by an external fixator. During his admission to the Traumatology plant he begins with hemoptysis.

He presented a previous history of treatment with anabolic steroids intermittently in the context of bodybuilding practice. The last cycle had been done four weeks before with the administration of methenolone acetate. The patient denied the use of drugs and any other type of medication.

The clinical began with hemoptotic sputum that occurred up to four times in 24 hours. The patient did not report dyspnea and he was eupneic at rest, but the saturation at the time of examination was
93\% without oxygen therapy, which amounted to $98 \%$ with oxygen therapy at $21 / \mathrm{min}$. He did not present other pathological findings in the examination.

The analytical controls showed a slight increase in creatinine levels $(1.25 \mathrm{mg} / \mathrm{dL})$ that was attributed to a prerenal cause and that responded to the administration of fluid therapy. There was no leukocytosis, although there was a discrete neutrophilia without eosinophilia. Hemoglobin levels were slightly decreased $(111 \mathrm{~g} / \mathrm{L})$ and both the platelets and the coagulation study were normal.

Studies of autoantibodies (ANA, ANCA, precipitins, immunoglobulin's and total IgE) were performed, with negative results.

A chest radiograph was performed showing bilateral diffuse alveolar infiltrates (Figure 1) and a chest CT scan that showed a ground glass pattern due to occupation of the air space that supported the suspicion of alveolar haemorrhage (Figure 2).

A bronchoscopy was performed in which the presence of bleeding remains was verified. When the bronchoalveolar lavage was performed, a blood-thinning fluid was obtained and sent for study.

The patient was treated with corticosteroids at high doses $(1 \mathrm{~g} / 24$ $\mathrm{h}$ ), maintaining them for five days and with progressive reduction of the dose. He presented a good response to treatment without returning to episodes of hemoptysis during admission.

*Corresponding author: Belen Gomez Rodriguez, Hospital Universitario Virgen del Rocio, Seville, Spain, Tel: 658196605; E-mail: bgrneumo@gmail.com

Received September 10, 2018; Accepted September 28, 2018; Published October 04, 2018

Citation: Rodríguez GB, Portal JAR (2018) Diffuse Alveolar Hemorrhage after Anabolic Steroid Use: A Case Report. J Pulm Respir Med 8: 473. doi: 10.4172/2161 105X.1000473

Copyright: (2018 Rodríguez GB, et al. This is an open-access article distributed under the terms of the Creative Commons Attribution License, which permits unrestricted use, distribution, and reproduction in any medium, provided the original author and source are credited. 


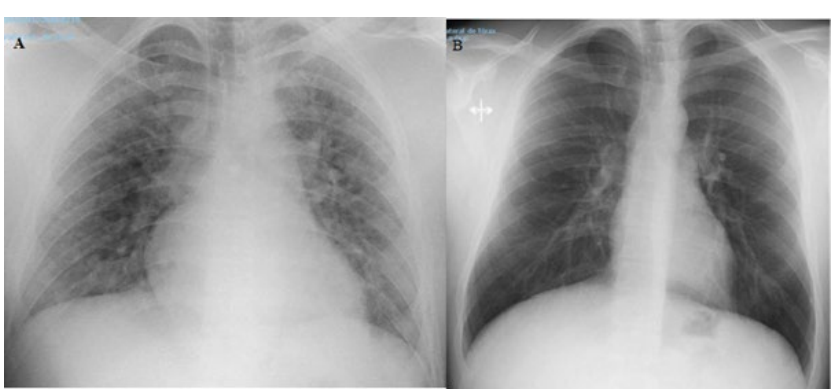

Figure 1: Rx thorax; (A) The first one was done during admission and shows a diffuse bilateral alveolar infiltrate. (B) The second was performed one month after the episode and shows a recovery of the lung parenchyma.
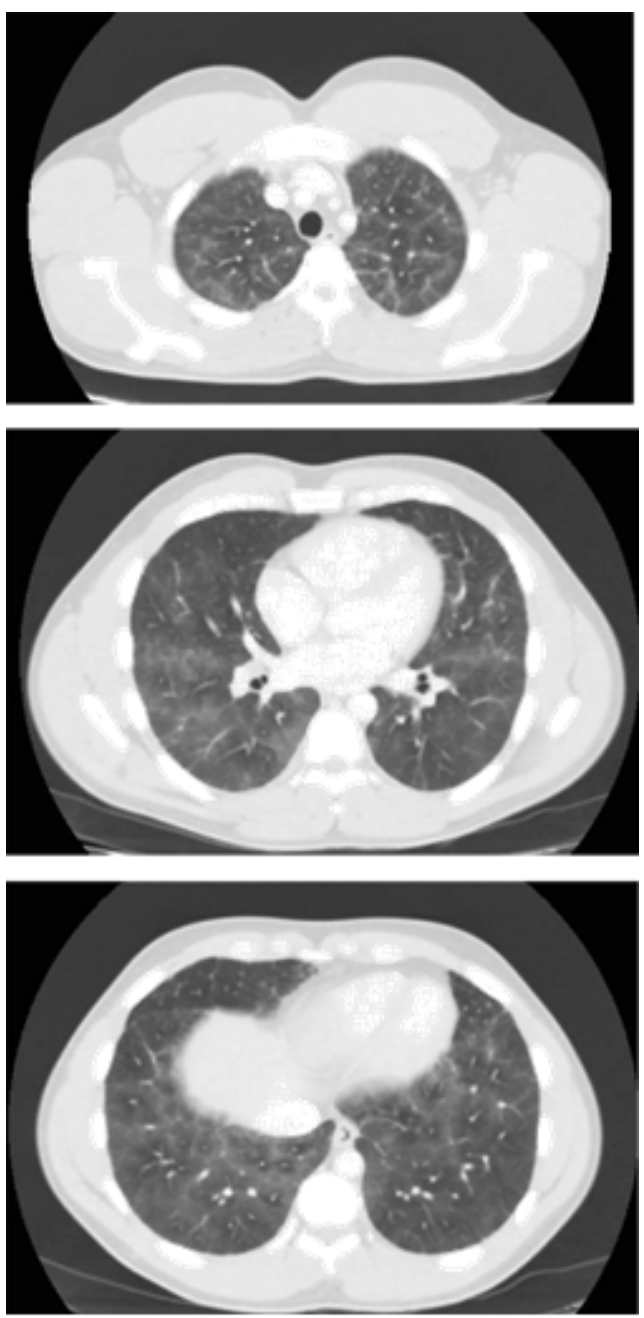

Figure 2: CT showing diffuse opacities in ground glass.

In the subsequent follow-up the patient has been asymptomatic, the radiographs of control carried out later have shown resolution of the alveolar infiltrates. Pulmonary function measured by spirometry and diffusion were normal. After admission, the patient has not been exposed to anabolic steroids again.

\section{Discussion}

Diffuse alveolar haemorrhage is characterized by the presence of hemoptysis, anaemia and diffuse alveolar infiltration. They are usually caused by vasculitis with involvement of small vessels and can be divided into 3 groups: pauciimunitaries, which present capillaritis and antineutrophil cytoplasmic antibodies, by immunological deposits and a very diverse third group that includes those idiopathic and caused by infections and drugs. It has a great associated morbidity and mortality if it is not diagnosed and treatment is started early [7].

For its diagnosis, it is necessary to carry out a correct clinical history and complementary tests that include laboratory tests (hemoglobin, platelets and coagulation), imaging tests (chest X-ray and CT) and serology with autoantibody studies [8,9].

There are two reported cases of alveolar haemorrhage associated with anabolic steroid abuse. There are also multiple cases of haemorrhage described in other organs after the use of anabolic steroids [1-6].

In this case, our patient was treated with high doses of corticosteroids for suspected vasculitis and given the high morbidity and mortality associated with this pathology. He was also told to avoid re-administering this medication, not returning to episodes of alveolar haemorrhage and with full recovery of the lung parenchyma as evidenced in the radiological and functional tests.

The pathophysiological causes of alveolar haemorrhage after anabolic steroids are not clear in these patients.

Both in the cases previously described and in that of our patient, the symptoms appeared weeks after the administration of the drug and remitted when avoiding exposure to it [7].

As for the imaging tests, the chest CT showed a diffuse alveolar infiltrate without peripheral predominance as in the previous cases [7].

\section{Conclusion}

1. We consider it important that, in the case of athletic patients who have undergone treatment with anabolic steroids and who have clinical and radiological findings compatible with diffuse alveolar haemorrhage, this medication should be suspected as a cause of it and should be withdrawn immediately.

\section{References}

1. Herlitz LC, Markowitz GS, Farris AB, Schwimmer JA Stokes MB et al. (2010) Development of focal segmental glomerulosclerosis after anabolic steroid abuse. J Am Soc Nephrol 21: 163-172.

2. Colburn S, Childers WK, Chacon A, Swailes A, Ahmed FM, et al. (2017) The cost of seeking an edge: Recurrent renal infarction in setting of recreational use of anabolic steroids. Ann Med Surg 14: 25-28.

3. Martin NM, Abu Dayyeh BK, Chung RT (2008) Anabolic steroid abuse causing recurrent hepatic adenomas and hemorrhage. World J Gastroenterol 14: 45734575.

4. Wysoczanski M, Rachko M, Bergmann SR (2008) Acute myocardial infarction in a young man using anabolic steroids. Angiol 59: 376-378.

5. Kennedy MC, Corrigan AB, Pilbeam ST (1993) Myocardial infarction and cerebral haemorrhage in a young body builder taking anabolic steroids. Aust N Z J Med 23: 713

6. Liljeqvist $S$, Helldén A, Bergman U, Söderberg M (2008) Pulmonary embolism associated with the use of anabolic steroids. Eur J Intern Med 19: 214-215.

7. Hvid-Jensen HS, Rasmussen F, Bendstrup E (2016) Pulmonary hemorrhage following anabolic agent abuse: Two cases. Respir Med Case Reports 18: 4547.

8. Council on Scientific Affairs (1990) Medical and nonmedical uses of anabolicandrogenic steriods. JAMA 264: 2923-2927.

9. Martín-Suñé N, Ríos-Blanco JJ (2012) Afectación pulmonar de las vasculitis Arch Bronconeumol 48: 410-418. 